\title{
CAPACIDADE PARA TOMADA DE DECISÃO EM IDOSOS INSTITUCIONALIZADOS E NÃO INSTITUCIONALIZADOS
}

\author{
Rúbia Garcia Deon ${ }^{1}$ \\ José Roberto Goldim²
}

resumo

Indivíduo autônomo é aquele que delibera sua vontade livremente de acordo com seus valores e crenças de vida. $\bigcirc$ presente artigo teve como objetivo avaliar a capacidade para a tomada de decisão em idosos institucionalizados e não institucionalizados. Trata-se de um estudo transversal, realizado em pessoas com mais de 60 anos de idade, em Instituições de Longa Permanência para Idosos e em Estratégias de Saúde da Família de Santa Cruz do Sul/RS. Para a coleta de dados, foi utilizado o instrumento de Desenvolvimento Psicológico-Moral. A análise dos resultados foi realizada pelos testes qui-quadrado e correlação linear, utilizando o programa SPSS 17.0. A significância estatística foi definida como $p<0.05$. Foram avaliados 552 idosos, sendo 276 institucionalizados e 276 não instituciona-

1 Graduada em Nutrição. Doutora em Gerontologia Biomédica. Docente no Curso de Nutrição da Universidade Integrada do Alto Uruguai e das Missões (URI). E-mail: rubiadeon@yahoo.com.br

2 Graduada em Biologia, Doutor em Medicina: Clínica Médica. Docente do Programa de Pós- Graduação em Gerontologia Biomédica/PUCRS. Atua no Laboratório de Pesquisa em Bioética e Ética na Ciência/HCPA. E-mail: jose.goldim@pucrs.br 
lizados, com idade média de 76,83 anos $\pm 7,36$ anos. A maioria eram mulheres, 306 (55,4\%), aposentados, 493 (89,3\%) e tinham o ensino fundamental, 228 (11,6\%). A classificação do Desenvolvimento Psicológico-Moral dos participantes se distribuiu entre as fases Conformista, Conscienciosa e Autônoma. Houve associação estatisticamente significativa desta variável com estar institucionalizado ou não ( $p=0,0001)$ e com o sexo ( $p=0,006)$. Mas, não foram observadas associações estatisticamente significativas com a idade, a escolaridade e a condição de ter ou não trabalhado fora de casa. Esta pesquisa demonstrou que todos os idosos tinham capacidade para a tomada de decisão, mesmo aqueles residentes em Instituições de Longa Permanência para Idosos. Desta forma, é preciso reconhecer os indivíduos nesta faixa etária como seres autônomos e sujeitos das próprias vontades.

palavras-chave

Idoso. Institucionalização. Desenvolvimento Psicológico-Moral.

\section{Introdução}

Um dos fenômenos de maior impacto no início do século XXI foi o aumento do número de pessoas idosas na população mundial, observado em países desenvolvidos e naqueles em desenvolvimento, como é o caso do Brasil (SAQUETTO et al., 2013). Atualmente, existem, no país, 11 milhões de pessoas com mais de 60 anos. As projeções indicam que, em 2025, serão 32 milhões de idosos, colocando o Brasil em sexto lugar mundial na quantidade de pessoas nesta faixa etária (IBGE, 2010).

A causa principal desse crescimento acelerado é a mudança no perfil epidemiológico do país, resultante do avanço da tecnologia e da melhoria nas condições de saúde. É possível observar uma redução de nascimentos e da mortalidade precoce, acompanhados pelo aumento no tempo de sobrevida de pessoas com doenças crônicas (CUNHA et al., 2012).

O rápido aumento de pessoas nesta faixa etária no Brasil gerou a demanda e o surgimento de Instituições de Longa Permanência para Idosos (ILPI) (CORNÉLIO; GODOY, 2013). Estas são autorizadas e regulamentadas por meio de legislação (BRASIL, 2005). Atualmente, estima-se que $1 \%$ dos brasileiros com 
mais de 60 anos residam nesses locais, totalizando, aproximadamente, 84.000 pessoas, e, devido ao número crescente de idosos, a tendência é o aumento de instituições no país (CAMARANO; KANSO, 2010).

O envelhecimento é um processo natural e irreversível. Faz parte do ciclo da vida do ser humano e ocorre de forma diferenciada em cada pessoa. É responsável por uma série de alterações, biológicas e sociais, que demandam atenção especial para assegurar as especificidades dessa população (CAMACHO; SANTOS, 2013).

Dentro deste ponto de vista, muitas pessoas partem do pressuposto de que o idoso é um ser vulnerável e, portanto, incapaz de tomar decisão sobre sua própria vida (SCHUMACHER; PUTTINI; NOJIMOTO, 2013). Outros acreditam que o idoso perde, de fato, a aptidão para escolher o que é melhor para si mesmo conforme avança o decurso da vida (CUNHA et al., 2012). Apesar de ser uma questão ainda controversa, existem algumas evidências em sentido contrário (HESS; QUEEN; ENNIS, 2013).

A privação da autonomia pode ser ainda mais eminente quando o idoso está institucionalizado (RAMOS et al., 2012). A perda da identidade pessoal e a rotina imposta pelas ILPI reduzem a perspectiva do idoso poder exercer a sua vontade (DUARTE, 2014). Assim, justifica-se a importância de investigar estes aspectos, sob diferentes óticas, entre a população idosa. O presente artigo teve, por objetivo, avaliar a capacidade para a tomada de decisão em idosos institucionalizados e não institucionalizados.

\section{Metodologia}

Trata-se de um estudo transversal, realizado com idosos, de ambos os sexos, do município de Santa Cruz do Sul/RS. Os critérios de inclusão foram ter mais de 60 anos, residir no município onde foi realizada a pesquisa e ter condições de responder ao instrumento. O critério de exclusão foi estar hospitalizado no momento da coleta de dados.

A pesquisa foi realizada com o conhecimento da Secretaria Municipal da Saúde e com o apoio do Conselho Municipal do Idoso, após a aprovação da Comissão Científica e do Comitê de Ética em Pesquisa (CEP) do Instituto de Geriatria e Gerontologia da PUCRS (CAAE 11986413.5.0000.5336). Todos os participantes receberam explicações claras e concisas em relação à pesquisa, procedendo à assinatura do Termo de Consentimento Livre e Esclarecido (TCLE). 
A coleta de dados ocorreu de forma auto aplicada assistida, no período correspondente entre junho de 2013 e maio de 2014. Existem 17 Instituições de Longa Permanência para Idosos (ILPI) cadastradas na Secretaria Municipal de Saúde de Santa Cruz do Sul/RS. Todos os idosos residentes nessas instituições que se enquadravam nos critérios de inclusão foram convidados para participar desta pesquisa. Os que aceitaram documentaram a sua vontade por meio da assinatura do TCLE. Foram incluídos 276 idosos institucionalizados. O grupo comparativo foi composto por idosos não institucionalizados, também residentes no município de Santa Cruz do Sul, pareados por critérios de sexo, idade e escolaridade. A escolha por utilizar o mesmo município para a obtenção destes dados teve como finalidade reduzir fatores de confusão, especialmente os culturais. Foram selecionados 276 idosos cadastrados nas Estratégias de Saúde da Família (ESFs) de diferentes regiões daquele município.

O instrumento utilizado foi o de Desenvolvimento Psicológico-Moral, elaborado e validado por Souza (1968). Baseado na perspectiva desenvolvida por Loevinger e Wessler, este método entende que o ego do indivíduo se desenvolve pela integração das estruturas de cada pessoa (LOEVINGER; WESSLER, 1970). Esse instrumento é composto por um conjunto de 30 itens de fácil compreensão, dos quais o participante deve escolher os sete mais significativos. Essa seleção permite enquadrar o idoso em uma das sete fases do Desenvolvimento Psicológico-Moral (SOUZA, 1968).

O somatório da pontuação pode classificar o idoso como Pré-social, com pontuação entre 0,1 e 1,0, corresponde ao início do desenvolvimento; Impulsivo, pontuação de 1,1 a 2,0, as decisões são tomadas a partir do desejo, não considerando as informações; Oportunista, pontuação entre 2,1 e 3,0, há valorização dos desejos e dos subsídios para que eles sejam atingidos; Conformista, pontuação de 3,1 a 4,0, no qual as crenças do indivíduo se sobrepõem aos desejos; Consciencioso, pontuação entre 4,1 e 5,0, tem capacidade de tomar decisão de forma autônoma, considerando seus os desejos e crenças, mas ainda é passível de constrangimento; Autônomo, pontuação de 5,1 a 6,0, capaz de tomar decisões livres de constrangimento, de modo independente; Integrado, pontuação entre 6,1 e 7,0, enxerga-se como parte de um todo e possui compreensão de sua interdependência (LOEVINGER; WESSLER, 1970). Os resultados foram tabulados e analisados com auxílio do programa SPSS 17.0 e a significância estatística foi definida como $p<0.05$. Os testes utilizados para as associações entre os dados foram o qui-quadrado e a correlação linear. 
e 276 não institucionalizados, pareados individualmente por sexo, idade e escolaridade. Participaram do estudo 306 (55,4\%) mulheres e 246 (44,6\%) homens. A idade mínima foi de 63 anos e a máxima, 99 anos, com média de 76,83 anos $\pm 7,36$ anos.

Quanto à profissão, 493 (89,3\%) eram aposentados e 57 (10,7\%) nunca trabalharam fora profissionalmente. Em relação à escolaridade, $26(1,3 \%)$ eram analfabetos, $274(13,9 \%)$ tinham o ensino fundamental, 228 (11,6\%) haviam concluído o ensino médio e $24(1,2 \%)$ possuíam ensino superior.

Quanto ao Desenvolvimento Psicológico-Moral, os grupos de indivíduos se enquadraram nas fases Conformista, Conscienciosa e Autônoma. Todos os 552 idosos mantinham a capacidade para a tomada de decisão preservada, ou seja, estavam aptos a fazer suas escolhas de acordo com o seu melhor interesse.

A comparação entre o Desenvolvimento Psicológico-Moral e a institucionalização apresentou uma associação estatisticamente significativa $(X 2=18,62$; $\mathrm{p}=0,0001)$. A maioria de ambos os grupos se localizou na fase Conscienciosa, sendo $67,4 \%$ dos participantes institucionalizados e $82,6 \%$ dos participantes não institucionalizados, conforme pode ser visualizado na Tabela 1.

Tabela 1 - Comparação entre Desenvolvimento Psicológico-Moral e institucionalização.

\begin{tabular}{l|c|c|c}
\hline \multirow{2}{*}{$\begin{array}{c}\text { Desenvolvimento } \\
\text { Psicológico-Moral }\end{array}$} & \multicolumn{2}{|c|}{ Institucionalização } & \multirow{2}{*}{ Total } \\
\cline { 2 - 3 } & Sim & Não & \\
\hline Conformista & $50(18,1 \%)$ & $21(7,6 \%)$ & $69(12,9 \%)$ \\
\hline Consciencioso & $186(67,4 \%)$ & $228(82,6 \%)$ & $414(75 \%)$ \\
\hline Autônomo & $40(14,5 \%)$ & $27(9,8 \%)$ & $67(12,1 \%)$ \\
\hline Total & $276(50,0 \%)$ & $276(50,0 \%)$ & $552(100 \%)$ \\
\hline
\end{tabular}

Fonte: Tabela elabora pelos autores.

A associação do Desenvolvimento Psicológico-Moral com dados sóciodemográficos foi estatisticamente significativa com o sexo dos participantes $\left(X^{2}=10,35 ; P=0,006\right)$, sendo que os homens tiveram maior frequência na fase 
Autônoma (58,2\%) e as mulheres, nas fases Conformista (55,3\%) e Conscienciosa $(69,0 \%)$. Não houve associações estatisticamente significativas entre a idade, a escolaridade e a condição dos participantes terem ou não trabalhado fora de casa, conforme pode ser observado na Tabela 2.

Tabela 2 - Comparação entre Desenvolvimento Psicológico-Moral e variáveis sociodemográficas.

\begin{tabular}{l|c|c|c}
\hline \multicolumn{1}{c|}{$\begin{array}{c}\text { Comparação do Desenvolvimento } \\
\text { Psicológico-Moral com }\end{array}$} & $\begin{array}{c}\text { Tipo de } \\
\text { teste }\end{array}$ & Valor obtido & P \\
\hline Idade & F & 0,482 & 0,618 (NS) \\
\hline Escolaridade & F & 0,404 & 0,766 (NS) \\
\hline Atividade profissional & $X^{2}$ & 5,461 & 0,065 (NS) \\
\hline Sexo & $X^{2}$ & 10,350 & $0,006(\mathrm{~S})$ \\
\hline
\end{tabular}

Fonte: Tabela elabora pelos autores.

Um dos 30 itens que compõem o instrumento para avaliar o Desenvolvimento Psicológico-Moral é a Autonomia, que foi selecionado por $29,2 \%$ dos participantes do estudo. Avaliando este item, de forma isolada, foi possível verificar que a maioria dos participantes que selecionaram este item como uma preocupação mais frequente com relação a si mesmo era do grupo institucionalizado (57,1\%). Esta associação foi estatisticamente significativa $(\mathrm{X} 2=4,64 ; \mathrm{P}<0,03)$.

\section{Discussão}

O envelhecimento traz, em seu contexto, inúmeras discussões de grande relevância, como a autonomia e a institucionalização (OLIVEIRA; ALVES, 2010). A importância destas questões também foi especificada na Política Nacional de Saúde da Pessoa Idosa, no intuito de manter o atendimento das necessidades pertinentes a pessoas nesta faixa etária (BRASIL, 2006).

O conceito de saúde no processo de envelhecimento se traduz mais pela condição de autonomia do idoso do que pela presença ou ausência de doença (SILVA et al., 2012). No presente estudo, todos os idosos apresentaram condições plenas para a tomada de decisão de acordo com o seu melhor interesse. 
Observa-se que a sociedade, muitas vezes, julga os idosos de forma equivocada, como indivíduos submissos e que possuem compreensão diminuída (ALMEIDA, 2010). A pessoa com mais de 60 anos de idade é considerada um ser frágil, incapaz e desconhecedor, portanto, do que é melhor para si (FERNANDES; GARCIA, 2010). A partir destes resultados, pode ser observado que a capacidade para tomada de decisão não está relacionada apenas com o avanço da idade, pois ela é particular de cada pessoa. A capacidade para tomada de decisão é um processo resultante de uma construção individual durante toda a vida (BAJOTTO; GOLDIM, 2011).

Devido à complexidade deste processo, não é possível estabelecer uma idade limite para a perda da capacidade para tomada de decisão (MEIRELES et al., 2012). É importante a construção de uma relação com o idoso livre de perspectivas preconceituosas. Isto poderá possibilitar o exercício da autonomia pelo idoso, ou seja, o poder de decisão sobre si mesmo, para assumir o controle de sua própria vida (CUNHA et al., 2012).

Os resultados da presente pesquisa reiteram estas constatações, previamente apresentadas. Todos os 552 idosos avaliados, tanto os institucionalizados como os não institucionalizados, tinham níveis de Desenvolvimento Psicológico-Moral compatíveis com a capacidade para a tomada de decisão no seu melhor interesse. Estes dados reiteram, igualmente, os já verificados em uma amostra de 670 idosos de grupos de terceira idade de Erechim/RS (CELICH; GOLDIM, 2010).

Vale ressaltar que foi verificada uma associação estatisticamente significativa entre a preocupação pontual com relação ao item Autonomia e a institucionalização. Esta maior preocupação pode se originar, pelo menos em parte, ao fato da liberdade, muitas vezes, ser restringida às ILPI (SAQUETTO et al., 2013). Os desejos e as vontades do idoso institucionalizado nem sempre são considerados (TAHAN; CARVALHO, 2010), assim como a sua aumentada dependência de terceiros para realizar as atividades da vida diária (FLORES et al., 2010).

Neste estudo, não foram verificadas associações estatisticamente significativas entre capacidade para tomada de decisão e as variáveis escolaridade e condição de ter ou não trabalhado fora de casa. Isto reitera a compreensão de que a capacidade para tomada de decisão é complexa, ou seja, é resultante de um somatório de múltiplas dimensões e não apenas de variáveis isoladas. Na presente amostra de idosos, mesmo aqueles que se enquadravam em condições tidas como desfavoráveis, tais como ter idade avançada, baixa escolaridade e sem nunca ter trabalhado fora de casa, foi verificada a preservação desta capacidade. 
Um aparente paradoxo poderia ser o achado resultante da comparação entre sexo e Desenvolvimento Psicológico-Moral. Os resultados desta pesquisa verificaram que o sexo masculino teve uma associação estatisticamente significativa com os níveis mais elevados de Desenvolvimento Psicológico-Moral. As diferenças de sexo, encontradas neste estudo, podem ser explicadas, pelo menos em parte, pelo processo histórico de construção e evolução da sociedade. As mulheres que hoje se encontram nessa faixa etária cresceram ainda baseadas no modelo patriarcal, com subordinação ao sexo masculino. A desproporção deste modo de vida pode ser um fator para determinar esta heterogeneidade observada na velhice (FERNANDES; GARCIA, 2010).

A maioria dos homens, que atualmente tem mais de 60 anos, eram estimulados para o trabalho fora de casa, enquanto que as mulheres, que hoje se encontram na mesma faixa etária, eram ensinadas para o trabalho doméstico (HEITOR; RODRIGUES; TAVARES, 2013). As oportunidades, os padrões sociais e culturais entre os sexos foram estabelecidos a partir de relações desiguais. Os homens tiveram maior liberdade para tomar suas próprias decisões durante toda a sua vida, enquanto que as mulheres foram reprimidas e tiveram o exercício da sua autonomia restringido, antes pelos pais e posteriormente por seus maridos (REIS; LÖBLER, 2012).

\section{Considerações finais}

Com base nos resultados obtidos na amostra estudada, foi possível verificar que todos os idosos que participaram desta pesquisa, institucionalizados e não institucionalizados, tinham o Desenvolvimento Psicológico-Moral compatível com a tomada de decisões conforme o seu melhor interesse. A idade, a escolaridade e a condição de ter ou não trabalhado fora de casa, não estão associadas com a capacidade para tomada de decisão, já o sexo masculino demonstrou uma associação estatisticamente significativa com os níveis mais elevados de Desenvolvimento Psicológico-Moral. Os participantes institucionalizados destacaram o item Autonomia como uma das preocupações mais frequentes com relação a si mesmos.

A preservação da capacidade para tomada de decisão é muito importante no envelhecimento. Todavia, o adequado entendimento desta necessidade ainda constitui um grande desafio. É fundamental avaliar esta questão de forma individual, e não associar exclusivamente à idade avançada. É preciso reconhecer o idoso como um ser autônomo e sujeito da sua própria vontade. 
abstract

Autonomous individual is the one who decides their will freely, according to their values and life beliefs. This paper aimed to evaluate the capacity for decision-making in elder institutionalized and non-institutionalized elderly. It is a cross-sectional study in people over 60 years of age, in long-stay institutions for the in the elderly and Family Health Strategies of Santa Cruz do Sul / RS. To collect data, it used the Psychological-Moral Development instrument. The analysis was performed by chi-square test and linear correlation, using the SPSS 17.0 program. Statistical significance was defined as $p<0.05$. We evaluated 552 elderly, 276 institutionalized and 276 non-institutionalized, with an average age of 76.83 years \pm 7.36 years. Most were women 306 (55.4\%), 493 retired (89.3\%) and 228 elementary school (11.6\%). The classification of Psychological-Moral Development of participants was distributed between Conformist stages, Conscientious and Autonomous. There was statistically significant association between this variable and institutionalized or not $(p=0.0001)$ and $\operatorname{sex}(p=0.006)$. But there was not statistically significant associations with age, education and the condition work outside the home. This research demonstrated that had capacity for decision making, even those living in long-stay institutions for the elderly. Thus, it is necessary to recognize individuals in this age group as autonomous beings and subjects of their own wills.

keywords

Elderly. Institutionalization. Psychological-Moral Development.

referências

ALMEIDA, Leonor Duarte de. Suscetibilidade: novo sentido para a vulnerabilidade. Revista Bioética, Brasilia, v. 18, n. 3, p. 537-548, 2010.

BAJOTTO, Aletheia Peters; GOLDIM, José Roberto. Avaliação da qualidade de vida e tomada de decisão em idosos participantes de grupos socioterápicos da cidade de Arroio do Meio, RS, Brasil. Revista Brasileira de Geriatria e Gerontologia, Rio de Janeiro, v. 14, n. 4, p. 753-761, out./dez. 2011. 
BRASIL. Ministério da Saúde. Portaria n. ${ }^{\circ}$ 2.528, de 01 de outubro 2006. Aprova a Política Nacional da Pessoa Idosa, 1 out. 2006, Brasília: Ministério da Saúde, 2006.

. Ministério da Saúde. Resolução n²83, de 26 de setembro 2005. Aprova o Regulamento Técnico para o funcionamento das Instituições de Longa permanência para Idosos, 26 set. 2005, Brasília: Ministério da Saúde, 2005.

CAMACHO, Alessandra Conceição Leite Funchal; SANTOS, Renata Costa. Revisión integrativa sobre las cuestiones bioéticas relacionadas con el anciano: aspectos relevantes para la enfermería. Enfermería Global, Murcia, v. 12, n. 31, p. 387-387, jul. 2013.

CAMARANO, Ana Amélia; KANSO, Solange. As instituições de longa permanência para idosos no Brasil. Revista Brasileira de Estudos da População, Rio de Janeiro, v. 27, n. 1, p. 233-235, jan./jun. 2010.

CELICH, Kátia Lilian Sedrez et al. Envelhecimento com qualidade de vida: a percepção de idosos participantes de grupos de terceira idade. Revista Mineira de Enfermagem, Belo Horizonte, v. 14, n. 2, p. 226-232, abr./jun. 2010.

CORNÉLIO, Graziela Félix; GODOY, Ilda de. Perfil das instituições de longa permanência para idosos em uma cidade no estado de São Paulo. Revista Brasileira de Geriatria e Gerontologia, Rio de Janeiro, v. 16, n. 3, p. 559-568, jul./set. 2013.

CUNHA, Juliana Xavier Pinheiro da et al. Autonomia do idoso e suas implicações éticas na assistência de enfermagem. Saúde em Debate, Rio de Janeiro, v. 36, n. 95, p. 657-664, out./dez. 2012.

DUARTE, Lidiane Mendes Nazareno. O processo de institucionalização do idoso e as territorialidades: espaço como lugar? Estudos Interdisciplinares sobre o Envelhecimento, Porto Alegre, v. 19, n. 1, p. 201-217, abr. 2014

FERNANDES, Maria das Graças Melo; GARCIA, Loreley Gomes. O sentido da velhice para homens e mulheres idosos. Saúde e Sociedade, São Paulo, v. 19, n. 4, p. 771-783, out./dez. 2010

FLORES, Gisela Cataldi et al. Cuidado intergeracional com o idoso: autonomia do idoso e presença do cuidador. Revista Gaúcha de Enfermagem, Porto Alegre, v. 31, n. 3, p. 467-474, set. 2010.

HEITOR, Sara Franco Diniz; RODRIGUES, Leiner Resende; TAVARES, Darlene Mara dos Santos. Prevalência da adequação à alimentação saudável e idosos residentes em zona rural. Texto \& Contexto: Enfermagem, Florianópolis, v. 22, n. 1, p. 79-88, jan./mar. 2013.

HESS, Tomas M.; QUEEN, Tara L.; ENNIS, Gilda E. Age and self-relevance effects on information search during decision making. The Journals of Gerontology Series B: Psychological Sciences and Social Sciences, Washington, v. 68, n. 5, p. 703-711, Sept. 2013.

INSTITUTO BRASILEIRO DE GEOGRAFIA E ESTATÍSTICA (IBGE). 2010. Disponível em: <http://www.ibge.gov.br>. Acesso em: 17 out. 2014

LOEVINGER, Jane; WESSLER, Ruth. Measuring ego development: Construction and use of a sentence completion test. San Francisco: Jossey-Bass, 1970. v. 1.

MEIRELES, Viviani Camboin et al. Autonomía y derecho a la información: contribuciones para la gestión del cuidado de ancianos hospitalizados. Ciencia y Enfermería, Concepción, v. 16, n. 2, p. 59-68, agosto 2010.

OLIVEIRA, Iglair Regis de; ALVES, Vicente Paulo. A pessoa idosa no contexto da Bioética: sua autonomia e capacidade de decidir sobre si mesma. Kairós Gerontologia, São Paulo, v. 13, n. 2, p. 91-98, nov. 2010

RAMOS, Luciana Junqueira et al. Ethical and nutrition issues in a sample of institutionalized and non-institutionalized elderly. Revista HCPA, Porto Alegre, v. 32, n. 2 , p. 223-226, 2012. 
REIS, Eliete dos; LÖBLER, Mauri Leodir. O processo decisório descrito pelo indivíduo e representado nos sistemas de apoio à decisão. Revista de Administração Contemporânea, Curitiba, v. 16, n. 3, p. 397-417, maio/jun. 2012.

SAQUETTO, Micheli et al. Aspectos bioéticos da autonomia do idoso. Revista Bioética, Brasília, v. 21, n. 3, p. 518-524, set./dez. 2013.

SCHUMACHER, Aluisio Almeida; PUTTINI, Rodolfo Franco; NOJIMOTO, Toshio. Vulnerabilidade, reconhecimento e saúde da pessoa idosa: autonomia intersubjetiva e justiça social. Saúde em Debate, Rio de Janeiro, v. 37, n. 97, p. 281-293, abr./jun. 2013.

SILVA, Roberto Jerônimo dos Santos et al. Prevalência e fatores associados à percepção negativa da saúde em pessoas idosas no Brasil. Revista Brasileira de Epidemiologia São Paulo, v. 15, n. 1, p. 49-62, mar. 2012

SOUZA, Edela Lanzer Pereira. Pesquisa sobre as fases evolutivas do ego. Boletim da Sociedade de Psicologia do Rio Grande do Sul, Porto Alegre, v. 3, n.7, p. 5-16, 1968

TAHAN, Jennifer; CARVALHO, Antonio Carlos Duarte de. Reflexões de idosos participantes de grupos de promoção de saúde acerca do envelhecimento e da qualidade de vida. Saúde e Sociedade, Paulo, v. 19, n. 4, p. 878-888, out./dez. 2010.

Recebido: 12/04/2015

Aceite Final: 18/08/2016 